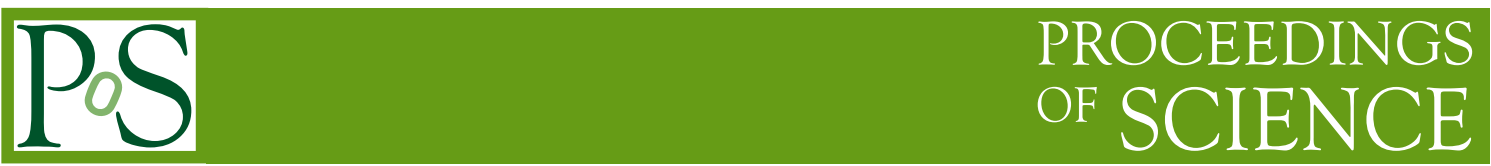

\title{
Progress towards vector boson pair production at NNLO*
}

\section{Dirk Rathlev University of Zurich}

E-mail: rathlev@physik.uzh.ch

\begin{abstract}
We present results for the first fully differential NNLO QCD computation of $Z \gamma$ production at hadron colliders. We find that the size of the NNLO corrections varies between $7 \%$ and $16 \%$ for typical LHC studies, depending on the applied cuts. We also observe that the purely loopinduced gluon initiated contribution - often included in NLO computations due to the large gluon luminosity at the LHC - constitutes less than $10 \%$ of the NNLO effect.
\end{abstract}

11th International Symposium on Radiative Corrections (Applications of Quantum Field Theory to Phenomenology)

22-27 September 2013

Lumley Castle Hotel, Durham, UK

\footnotetext{
${ }^{*}$ In collaboration with Massimiliano Grazzini, Stefan Kallweit and Alessandro Torre.

† Speaker.
} 


\section{Introduction}

Vector boson pair production processes $p p \rightarrow V V^{\prime}$, with $V, V^{\prime} \in\left\{\gamma, W^{ \pm}, Z\right\}$, play an important role for many SM and BSM studies at the LHC. All of these processes appear as irreducible background in SM or BSM Higgs studies, and direct measurements of these processes allow precise studies of the electroweak gauge structure of the standard model. Thus, obtaining detailed theoretical predictions for all vector boson pair production processes is of great importance. We give a short overview of the status of these calculations in the following. For diphoton production $p p \rightarrow \gamma \gamma$ a fully exclusive NNLO QCD computation exists [1]. For the next-to-simplest processes $p p \rightarrow W / Z \gamma$ (which have three particles in the final state after taking the decay of the heavy vector boson into account) the NLO QCD corrections [2], the gluon initiated loop-induced NNLO contribution [3] and the dominant electroweak corrections [4] are known. This talk reports on the first fully exclusive NNLO QCD computation of $p p \rightarrow Z \gamma$, which has been finished recently [5].

\section{The computation}

All necessary ingredients for the NNLO QCD computation are available: the real-virtual amplitudes have been computed in [6,7], the gluon induced NNLO contribution can be found in [3]. The Drell-Yan like final state radiation contribution to the two-loop corrections of the Born level process has been known for a long time [8] and the computation of the full amplitude has been completed recently [9]. Out of convenience, we use the one-loop QCD amplitude generator OpenLoops [10] interfaced with the Collier tensor integral library [11] to obtain all tree- and one-loop amplitudes needed in the calculation.

Even with all ingredients available, performing the NNLO calculation is still a highly nontrivial undertaking due to the presence of infrared singularities - both as explicit poles in the virtual amplitudes and as divergences of the phase space integration of the real corrections - at intermediate stages of the computation. These singularities are guaranteed to cancel out for all infrared safe observables after the combination of all contributions; however, as this is only possible after (part of) the phasespace integration has been carried out, a procedure is needed to achieve this in numerical calculations. The method we are going to use - the $q_{T}$-subtraction method [12] - has been successfully used for several NNLO computations of production processes of colorless final states [1,13].

The starting point of the $q_{T}$-subtraction method is the observation that as long as the transverse momentum $q_{T}$ of the final state system $F$ is non-vanishing, a (N)NLO computation of $p p \rightarrow F+X$ amounts to a (N)LO computation of $p p \rightarrow F+$ jet $+X$ :

$$
\left.\mathrm{d} \sigma_{(N) N L O}^{F}\right|_{q_{T} \neq 0}=\mathrm{d} \sigma_{(N) L O}^{F+j e t s} .
$$

Of course, this cross section is IR divergent in the limit $q_{T} \rightarrow 0$. However, the structure of this divergence is known from studies of transverse momentum resummation [14] and allows one to write down a universal (process independent) counterterm $\mathrm{d} \sigma^{C T}=\Sigma\left(q_{T} / Q\right) \otimes \mathrm{d} \sigma_{L O}$, where $Q \equiv m_{F}$ denotes the invariant mass of the final state system $F$. To obtain the full (N)NLO cross section, the missing piece of the $q_{T}=0$ contribution needs to be added. This piece contains the loop corrections 
to the Born level subprocess and can be written as $\mathscr{H}_{(N) N L O}^{F} \otimes \mathrm{d} \sigma_{L O}$. Adding everything together, one obtains

$$
\mathrm{d} \sigma_{(N) N L O}^{F}=\mathscr{H}_{(N) N L O}^{F} \otimes \mathrm{d} \sigma_{L O}+\left[\mathrm{d} \sigma_{(N) L O}^{F+j e t s}-\mathrm{d} \sigma_{(N) N L O}^{C T}\right] .
$$

The counterterm $\mathrm{d} \sigma_{(N) N L O}^{C T}$ is - up to a dependence on $\mathscr{H}_{(N) L O}^{F}$ - process independent and known explicitly. The NLO contribution to $\mathscr{H}^{F}$, denoted $\mathscr{H}^{(1) F}$, can be obtained from the one-loop amplitude of the Born level process via a process independent relation [15]. This relation has been generalized to all orders and has been explicitly evaluated up to the NNLO, i.e. up to $\mathscr{H}^{(2) F}$, in [16].

\section{Phenomenology of $Z \gamma$ production}

In the following, we present results for the production of a $Z \gamma$ final state system at full NNLO QCD accuracy, based on the calculation in Ref. [5]. We note that the notation $Z \gamma$ is misleading, as we do take all off-shell effects, interference between $Z$ and $\gamma$ intermediate states, and photon radiation from the final state into account. In fact, we consider the process $p p \rightarrow \ell^{+} \ell^{-} \gamma$, with $\ell=e, \mu$, without any approximations.

Processes with photons in the final state suffer from additional IR singularities when the photon becomes collinear to a final state quark. These singularities cancel once QCD fragmentation processes of the type $q \rightarrow \gamma+X$ are taken into account. However, these effects are non-perturbative in nature and have to be described by a poorly known photon fragmentation function. In addition, the $q_{T}$ subtraction method currently does not allow for non-colorless particles in the final state of the Born level subprocess, hence an implementation of photon fragmentation is not possible at the NNLO.

Experimentally, photons have to fulfill some kind of isolation requirement with respect to QCD final state particles. Usually, a hard cone isolation is applied, i.e. the condition for a photon to be isolated is

$$
\sum_{\delta<\delta_{0}} E_{T}^{\text {had }} \leq \varepsilon_{\gamma} E_{T}^{\gamma}
$$

where the left-hand side denotes the sum of all hadronic energy inside a cone of radius $\delta_{0}$ around the photon and this is compared to a fraction $\varepsilon_{\gamma}$ of the photon transverse energy $E_{T}^{\gamma}$. This isolation prescription removes most, but not all of the fragmentation contribution and still leads to IR divergences when the fragmentation contribution is neglected completely. An alternative isolation prescription, which removes the fragmentation contribution completely, is the so-called smooth cone isolation [17]. The corresponding criterion for isolated photons reads ${ }^{1}$

$$
\sum_{\delta^{\prime}<\delta} E_{T}^{h a d} \leq \varepsilon_{\gamma} E_{T}^{\gamma} \chi(\delta) \quad \text { for all } \quad \delta \leq \delta_{0}, \quad \chi(\delta)=\left(\frac{1-\cos (\delta)}{1-\cos \left(\delta_{0}\right)}\right)
$$

i.e. it consists of a family of cuts, which contains the hard cone isolation criterion Eq. (3.1) for $\delta=\delta_{0}$, but applies additional cuts on events with soft QCD activity very close to the photon.

\footnotetext{
${ }^{1}$ There is some freedom in choosing the function $\chi$. Its critical properties are smoothness, $\chi\left(\delta_{0}\right)=1$ and $\chi(\delta) \rightarrow 0$ for $\delta \rightarrow 0$ sufficiently fast.
} 


\begin{tabular}{l|l|l|l|l|l} 
& & LO & NLO & NNLO & measurement \\
\hline ATLAS setup & $\sigma[\mathrm{pb}]$ & $0.851(1)$ & $1.226(1)$ & $1.321(3)$ & $1.31(12)$ \\
$p_{T}^{\gamma}>15 \mathrm{GeV}$ & rel. correction & & $44 \%$ & $8 \%$ & \\
\hline ATLAS setup & $\sigma[\mathrm{fb}]$ & $77.48(6)$ & $132.90(7)$ & $153.3(5)$ & \\
$p_{T}^{\gamma}>40 \mathrm{GeV}$ & rel. correction & & $72 \%$ & $16 \%$ & \\
\hline \multirow{2}{*}{ CMS setup } & $\sigma[\mathrm{pb}]$ & $1.334(1)$ & $1.891(1)$ & $2.021(6)$ & \\
& rel. correction & & $42 \%$ & $7 \%$ &
\end{tabular}

Table 1: Total cross sections and relative sizes of the higher order corrections for $p p \rightarrow \ell^{+} \ell^{-} \gamma$ with three different sets of cuts at LO, NLO and NNLO. The result of the ATLAS measurement in the first setup is also shown.

Recent studies [21] have shown that the difference in cross sections obtained with hard cone and with smooth cone isolation is usually at the percent level even for moderate values of $\delta_{0}$ and $\varepsilon_{\gamma}$ and vanishes almost completely if the isolation parameters are lowered, i.e. the isolation is tightened. We verified for the setups we are going to consider in the following that the impact of using smooth cone isolation instead of hard cone isolation amounts to a difference of about two percent, thus allowing a direct comparison of our results with experimental data, even though we will be using the smooth cone isolation.

We use the setup employed by the ATLAS collaboration in one of their recent studies [18] of $\ell^{+} \ell^{-} \gamma$ production to illustrate some numerical results in the following. The set of cuts consists of transverse momentum and rapidity cuts on the final state photon $\left(p_{T}^{\gamma}>15 \mathrm{GeV},\left|\eta^{\gamma}\right|<2.37\right)$ and on the final state leptons $\left(p_{T}^{\ell}>25 \mathrm{GeV},\left|\eta^{\ell}\right|<2.47\right.$ ). In addition, there is a requirement on the invariant mass of the lepton pair $\left(m_{\ell \ell}>40 \mathrm{GeV}\right)$ and a lepton photon isolation criterion $(\Delta R(\ell, \gamma)>0.7)$. Jets are clustered with the anti- $k_{T}$ algorithm with radius $D=0.4$ and are required to fulfill $E_{T}^{\text {jet }}>30 \mathrm{GeV}$ and $\left|\eta^{j e t}\right|<4.4$. Events where a jet is too close to either the photon or one of the leptons are discarded $(\Delta R(\ell / \gamma, j e t)>0.3)$. ATLAS uses a hard cone isolation with parameters $\delta_{0}=0.4$ and $\varepsilon_{\gamma}=0.5$. In our study we apply a smooth cone isolation with the same parameters, which at NLO results in a difference of less than $2 \%$ compared to the MCFM NLO prediction used by ATLAS. The central values of the scales are set to $\mu_{R}=\mu_{F}=\sqrt{m_{Z}^{2}+\left(p_{T}^{\gamma}\right)^{2}}$. For the PDF sets we use MSTW2008, with LO, NLO and NNLO evolution in the LO, NLO and NNLO results respectively [19].

Table 3 shows the total cross sections at LO, NLO and NNLO for the ATLAS setup described above. We also show results for a slightly different setup also used by ATLAS, in which the photon transverse momentum cut is $p_{T}^{\gamma}>40 \mathrm{GeV}$, and for a setup used by CMS [20], which has slightly different cuts $\left(p_{T}^{\gamma}>15 \mathrm{GeV},\left|\eta^{\gamma}\right|<2.5, p_{T}^{\ell}>20 \mathrm{GeV},\left|\eta^{\ell}\right|<2.5, m_{\ell \ell}>50 \mathrm{GeV}, \Delta R(\ell, \gamma)>0.7\right.$, $\varepsilon_{\gamma}=0.05$ and $\left.\delta_{0}=0.15\right)$. For the first ATLAS setup $\left(p_{T}^{\gamma}>15 \mathrm{GeV}\right)$, we obtain $\sigma_{N L O}=1.226(1) \mathrm{pb}$ at NLO and $\sigma_{N N L O}=1.321(3) \mathrm{pb}$ at NNLO (errors are pure Monte Carlo errors), which can be compared to the measured cross section of $\sigma=1.31(12) \mathrm{pb}$. We observe that the NNLO/NLO ratio strongly depends on the setup and varies between 1.07 and 1.16 .

The $q_{T}$-subtraction method employs a non-local counterterm and thus a phase space regulating cut on $q_{T} / Q$, where $q_{T}$ and $Q$ are the transverse momentum and the invariant mass of the $\ell^{+} \ell^{-} \gamma$ 


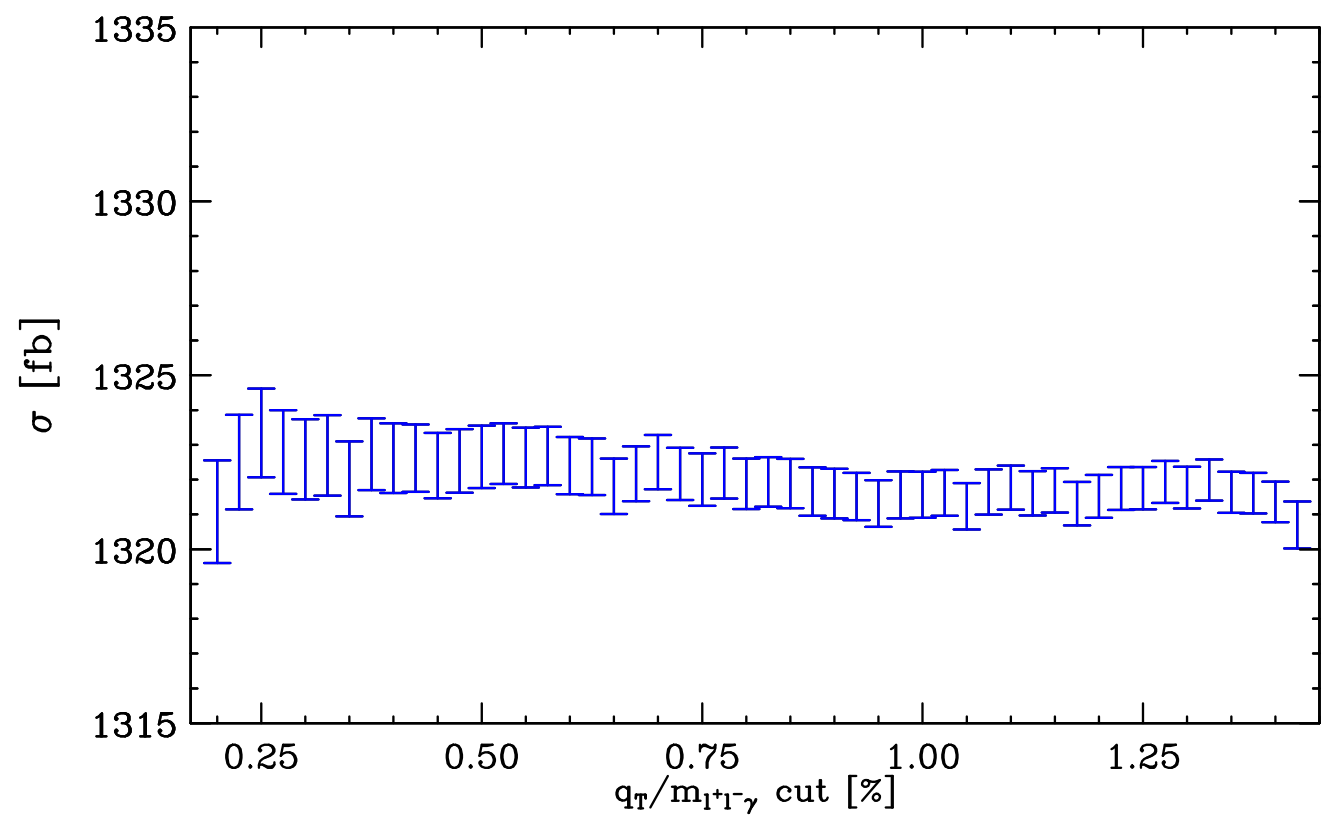

Figure 1: Dependence of the total cross section in the ATLAS $p_{T}^{\gamma}>15 \mathrm{GeV}$ setup on the regularization cut on $q_{T} / Q$.

final state system respectively, has to be introduced. The independence of the final result on this cut, varied in a reasonable region, constitutes a strong check on the correctness of the calculation. Fig. 1 shows the total cross section obtained with the first ATLAS setup as a function of the $q_{T} / Q$ cut. We observe that the result is - inside statistical uncertainties - completely independent of the cut value in the range considered.

It turns out that there is an accidental cancellation of the scale dependence if both scales are varied symmetrically (i.e. if we consider $\mu_{R}=a \mu_{0}=\mu_{F}$ for a central scale $\mu_{0}$ and $a \in[0.5,2]$ ), which leads to an almost vanishing scale dependence already at NLO. It has been argued in [2] that such a scale variation does not give a reliable estimate of the size of missing higher order corrections and it has been proposed to vary the scales antisymmetrically, i.e. setting $\mu_{R}=a \mu_{0}=a^{2} \mu_{F}$. Doing this, we obtain a scale uncertainty of ${ }_{-5 \%}^{+4 \%}$ at NLO and of ${ }_{-2 \%}^{+2 \%}$ at NNLO. The full dependence can be found in Fig. 2.

Fig. 3 shows the invariant mass distribution $\mathrm{d} \sigma / \mathrm{d} m_{\ell^{+} \ell^{-}}$at LO, NLO and NNLO as well as the differential NNLO/NLO ratio. The impact of the NNLO corrections is small in the region where the cross section is largest, explaining the moderate overall NNLO/NLO ratio, but the NNLO corrections grow more important in the high invariant mass tail of the distribution. Also shown is the purely loop-induced gluon initiated contribution, which, though formally part of the NNLO, is often included in NLO computations as the large gluon luminosity at the LHC can potentially overcome the additional factor of $\alpha_{s}$. In the case of $Z \gamma$ production however, the $g g$ contribution only amounts to less than $1 \%$ of the total cross section and less than $10 \%$ to the NNLO corrections.

Fig. 4 shows the same invariant mass distribution for the second ATLAS setup with $p_{T}^{\gamma}>$ $40 \mathrm{GeV}$. Due to an implicit cut on $m_{\ell^{+} \ell^{-} \gamma}$ at $\sim 97 \mathrm{GeV}$ at LO, the region around the $Z$ peak only 


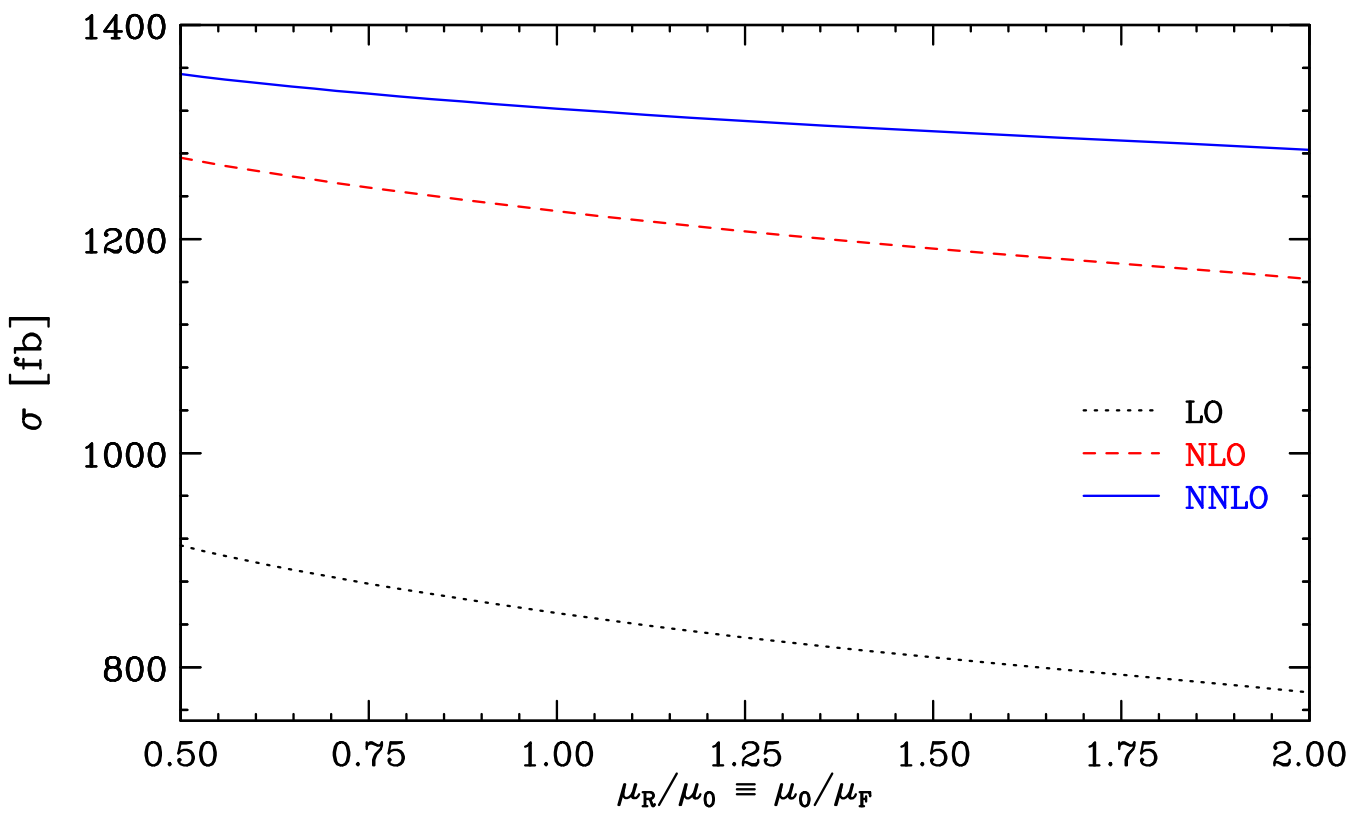

Figure 2: Scale dependence in the ATLAS $p_{T}^{\gamma}>15 \mathrm{GeV}$ setup when varying the scales antisymmetrically from $\mu_{0} / 2$ to $2 \mu_{0}$.

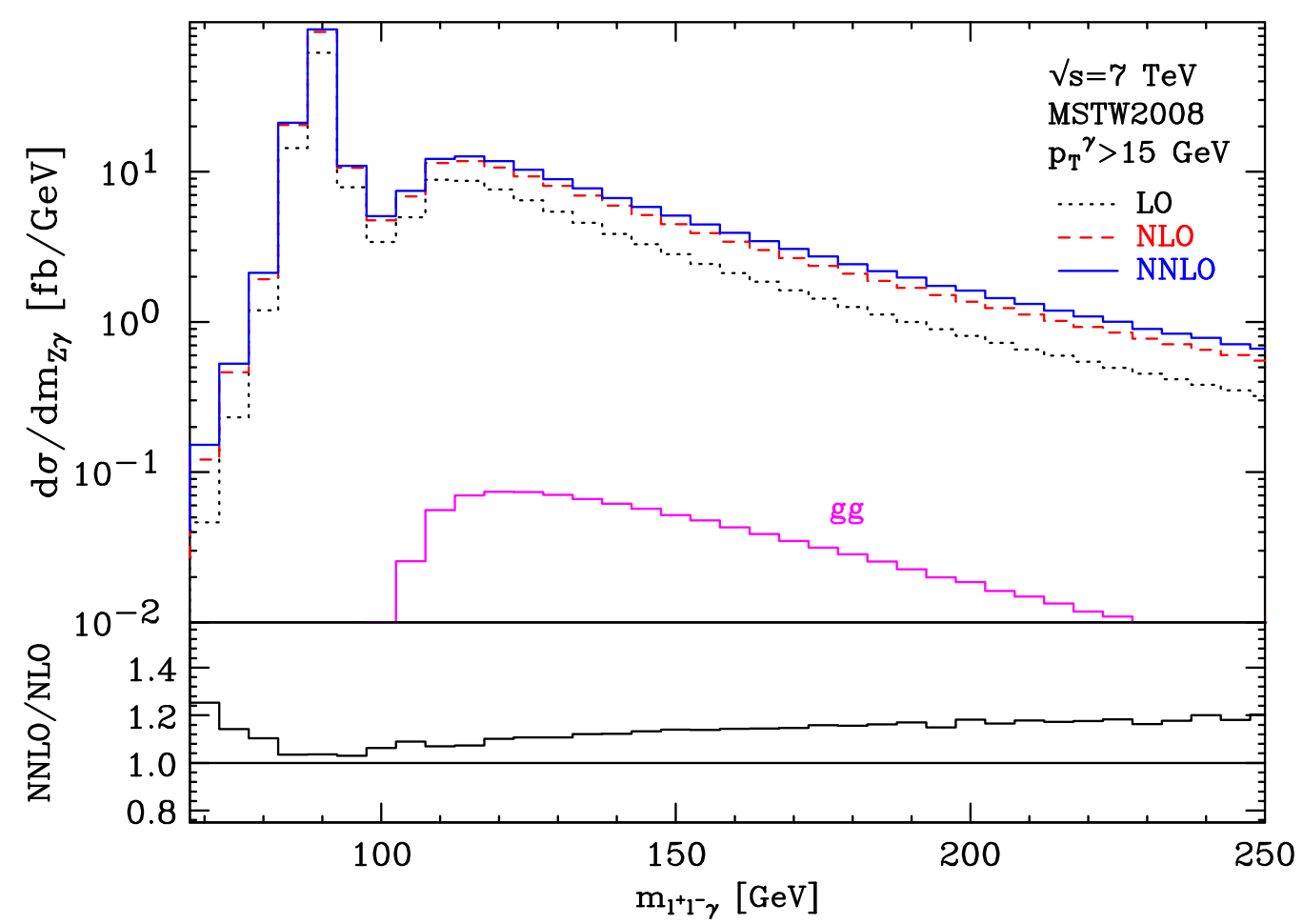

Figure 3: Invariant mass distribution of the $\ell^{+} \ell^{-} \gamma$ final state system at LO (dots), NLO (dashes) and NNLO (solid). The loop-induced $g g$ contribution is also shown. The lower panel shows the NNLO/NLO ratio. 


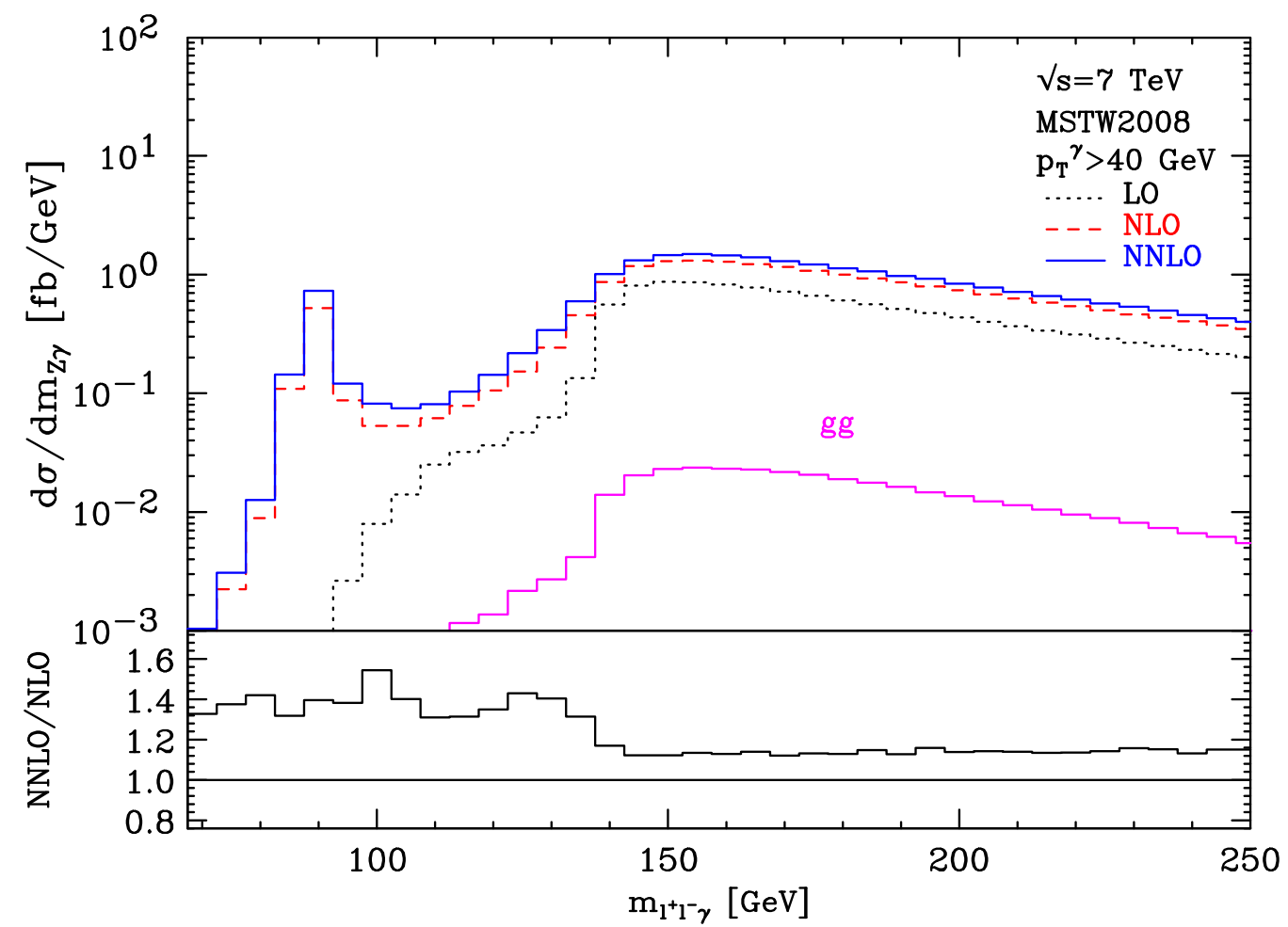

Figure 4: As Fig. 3, but with a $p_{T}^{\gamma}>40 \mathrm{GeV}$ cut.

opens up at NLO and thus the formally NLO result is in fact only LO in that region. This explains the enlarged NNLO/NLO ratio, as a significant part of the total cross section comes from a region where the NNLO computation is in fact only NLO.

\section{Summary}

We presented the first fully exclusive NNLO QCD calculation of $Z \gamma$ production. We studied different setups used by experimentalists in LHC analyses and found the size of the NNLO corrections to be between $7 \%$ and $16 \%$; the size of the corrections is not uniform over distributions and strongly depends on the set of cuts applied. We found the $g g$ loop-induced contribution to be very small and not to be a reliable estimate of the size of the full NNLO corrections. The next step in the computation of NNLO QCD corrections to vector boson pair production processes is the $p p \rightarrow W \gamma$ process, which is of comparable complexity as $Z \gamma$ production.

\section{References}

[1] S. Catani, L. Cieri, D. de Florian, G. Ferrera and M. Grazzini, Phys. Rev. Lett. 108 (2012) 072001.

[2] J. M. Campbell, R. K. Ellis and C. Williams, JHEP 1107 (2011) 018.

[3] L. Ametller, E. Gava, N. Paver and D. Treleani, Phys. Rev. D 32 (1985) 1699; J. J. van der Bij and E. W. N. Glover, Phys. Lett. B 206 (1988) 701. 
[4] W. Hollik and C. Meier, Phys. Lett. B 590 (2004) 69; E. Accomando, A. Denner and C. Meier, Eur. Phys. J. C 47 (2006) 125.

[5] M. Grazzini, S. Kallweit, D. Rathlev and A. Torre, report ZU-TH-21-13 (arXiv:1309.7000 [hep-ph]).

[6] Z. Bern, L. J. Dixon and D. A. Kosower, Nucl. Phys. B 513 (1998) 3.

[7] J. M. Campbell, H. B. Hartanto and C. Williams, JHEP 1211 (2012) 162.

[8] T. Matsuura, S. C. van der Marck and W. L. van Neerven, Nucl. Phys. B 319 (1989) 570.

[9] T. Gehrmann and L. Tancredi, JHEP 1202 (2012) 004.

[10] F. Cascioli, P. Maierhofer and S. Pozzorini, Phys. Rev. Lett. 108 (2012) 111601.

[11] A. Denner and S. Dittmaier, Nucl. Phys. B 734 (2006) 62, Nucl. Phys. B 844 (2011) 199.

[12] S. Catani and M. Grazzini, Phys. Rev. Lett. 98 (2007) 222002.

[13] S. Catani, L. Cieri, G. Ferrera, D. de Florian and M. Grazzini, Phys. Rev. Lett. 103 (2009) 082001; G. Ferrera, M. Grazzini and F. Tramontano, Phys. Rev. Lett. 107 (2011) 152003;

[14] G. Bozzi, S. Catani, D. de Florian and M. Grazzini, Nucl. Phys. B 737 (2006) 73.

[15] D. de Florian and M. Grazzini, Nucl. Phys. B 616 (2001) 247.

[16] S. Catani, L. Cieri, D. de Florian, G. Ferrera and M. Grazzini, [arXiv:1311.1654 [hep-ph]].

[17] S. Frixione, Phys. Lett. B 429 (1998) 369.

[18] G. Aad et al. [ATLAS Collaboration], Phys. Rev. D 87 (2013) 112003.

[19] A. D. Martin, W. J. Stirling, R. S. Thorne and G. Watt, Eur. Phys. J. C 63 (2009) 189.

[20] S. Chatrchyan et al. [CMS Collaboration], report CMS-EWK-11-009, CERN-PH-EP-2013-108, arXiv:1308.6832 [hep-ex].

[21] D. de Florian, talk given at the Workshop on Photon Physics and Simulation at Hadron Colliders, Paris, March 2012. 